\title{
Sub-Tenon Injection of Triamcinolone Acetonide for Choroidal Mass in Sarcoidosis: A Case Report
}

This article was published in the following Dove Press journal:

International Medical Case Reports Journal

\author{
Mihori Kita' \\ Yu Kagitani' \\ Sachiyo Hama' \\ Atsushi Azumi ${ }^{2}$ \\ 'Department of Ophthalmology, National \\ Hospital Organization Kyoto Medical \\ Center, Kyoto, Japan; ${ }^{2}$ Department of \\ Ophthalmology, Kobe Kaisei Hospital, \\ Kobe, Japan
}

Purpose: To report the clinical course of a sub-tenon injection of triamcinolone acetonide for treatment of an asymptomatic choroidal mass in sarcoidosis.

Methods: This was a retrospective, non-comparative interventional case study.

Results: A 36-year-old Japanese man with sarcoidosis presented with an asymptomatic choroidal mass lesion associated with subretinal fluid accumulation in his left eye, but without any other sign of intraocular inflammation in either of his eyes. He had started systemic steroid administration for thrombocytopenia 2 days prior to being examined. After a single sub-tenon injection of triamcinolone acetonide $(20 \mathrm{mg})$ the subretinal fluid was completely absorbed and the choroidal granuloma began to shrink in size. After the injection, the granuloma became a scar without any complication or recurrence over the next 34 months.

Conclusion: Sub-tenon injection of triamcinolone acetonide might be an effective treatment for choroidal granuloma in sarcoidosis.

Keywords: sarcoidosis, choroidal mass, choroidal granuloma, sub-tenon injection, triamcinolone acetonide, steroid

\section{Introduction}

The prevalence of ocular involvement in systemic sarcoidosis ranges from 13-79\% of patients. ${ }^{1-4}$ A posterior segment lesion without anterior segment involvement occurs in only $5 \%$ of patients with ocular sarcoidosis, with $12 \%$ of these patients developing choroidal granuloma. ${ }^{5-7}$ Thus, the presence of a choroidal granuloma, especially a solitary lesion, is a rare ocular sequela of sarcoidosis.

In the current study, we report the clinical course of a sub-tenon injection of triamcinolone acetonide for treatment of an asymptomatic solitary choroidal mass in a patient with biopsy-proven sarcoidosis.

\section{Case Report}

This study was approved by the Ethics Committee of the National Hospital Organization Kyoto Medical Center to publish the case details, and all procedures involved adhered to the tenets of the Declaration of Helsinki. Written informed consent for the procedure and participation in the study to have the case details and any accompanying images published was obtained from the patient after receiving an explanation of the therapeutic procedures.

A 36-year-old Japanese man with sarcoidosis was seen in July 2017 after referral by his internist. He was diagnosed with thrombocytopenic purpura and treated with $70 \mathrm{mg}$
Department of Ophthalmology, National Hospital Organization Kyoto Medical Center, I-I Mukaihata-cho, Fukakusa, Fushimi-ku, Kyoto-city, Kyoto 612-8555, Japan

Tel +8I-75-64I-9|6I

Fax +8I-75-643-4325

Email mihorik@kuhp.kyoto-u.ac.jp
International Medical Case Reports Journal 2021:14 33-38 
of prednisolone 2 days prior to his first eye examination for anemic retinopathy and sarcoidosis. At the start of the systemic steroid treatment, his platelet level was $4,000 / \mu \mathrm{L}$. This thrombocytopenia could be attributed to splenomegaly which is associated with the sarcoidosis.

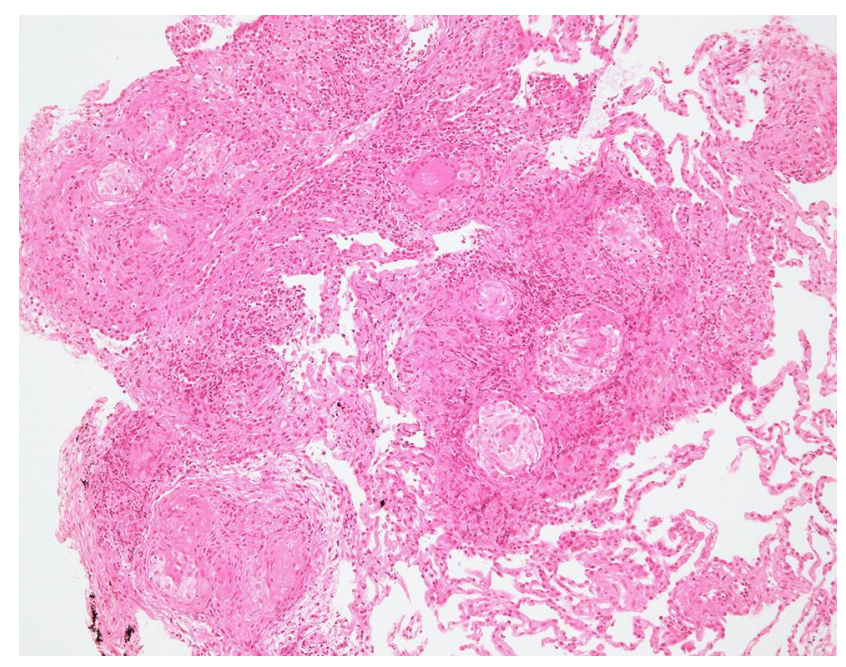

Figure I Histopathology image of bronchial biopsy. Hematoxylin and eosin stain (x|00). Non-necrotizing granulomatous reaction was observed, which was not inconsistent with sarcoidosis.
The patient's past history included a right cervical lymph node biopsy in March 2015, a left arm subcutaneous nodule biopsy in September 2015, and a transbronchial lung biopsy in January 2016, which revealed noncaseating epithelioid cell granuloma (Figure 1). Then the patient was diagnosed with biopsyproven sarcoidosis. The ACE level was 8.4-16.4 IU/L, within normal limits. Ocular examination in 2016 revealed no remarkable findings associated with sarcoidosis in either of his eyes. Recovery from the pulmonary sarcoid lesion occurred without systemic steroid administration. Although his serum lysozyme was elevated, serum angiotensin converting enzyme was low due to the administration of an oral angiotensin II receptor blocker for hypertension. Other routine blood investigations, including the serum level of calcium, eosinophils, erythrocyte sedimentation rate, and antibodies for toxoplasma, were all negative.

The patient was found to have an asymptomatic choroidal mass located at 1 disc diameter (DD) superior-temporal of the macula in his left eye. The protruded mass was almost round in shape, 1.5 DD in size, and white in color (Figure 2A). Ultrasound (US)-B scan demonstrated the presence of an
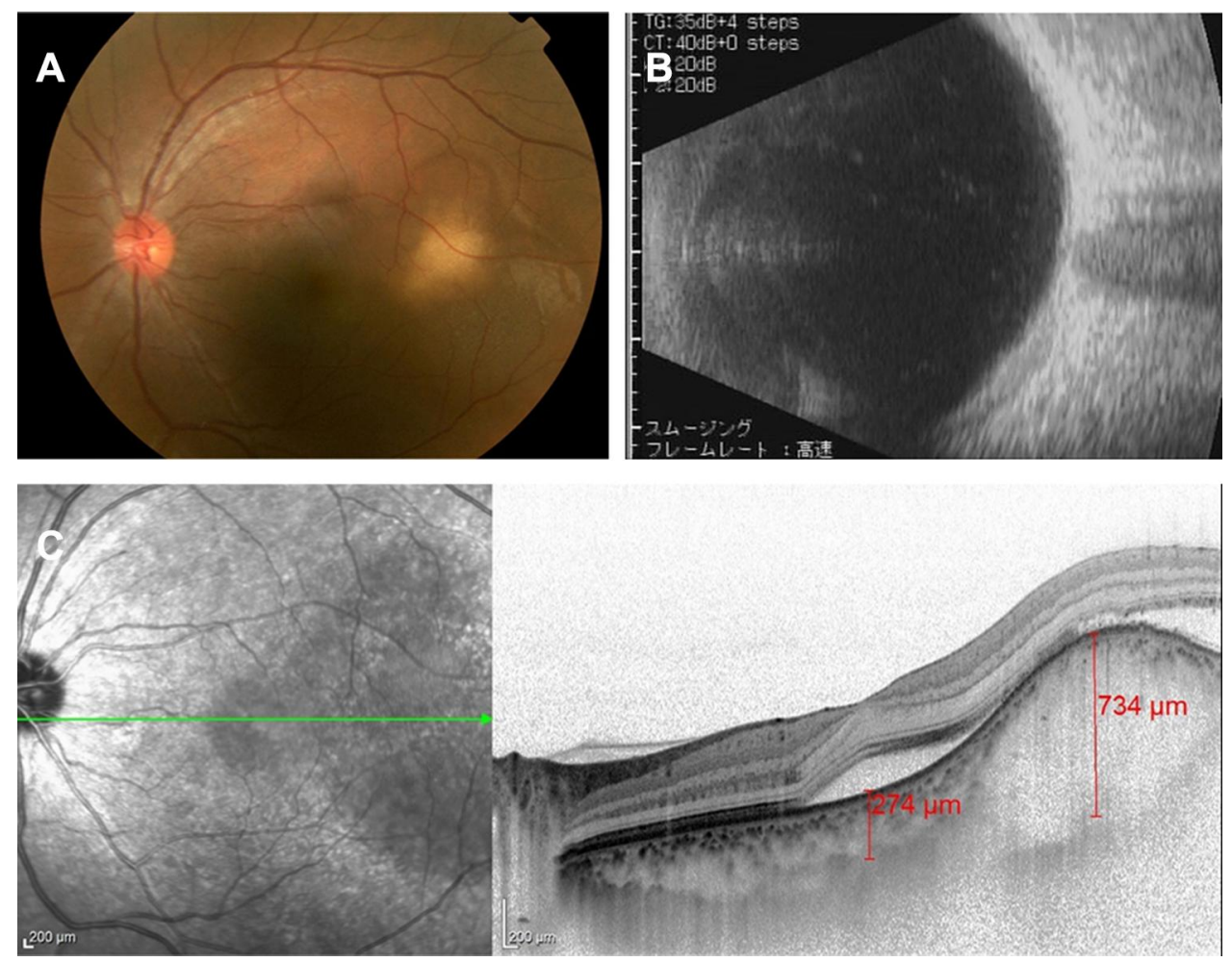

Figure 2 Ocular examination findings in his left eye at the first visit. (A) Fundus photograph shows a white choroidal mass at superior-temporal of the macula. (B) US-B scan showed no evidence of calcification within the mass. (C) EDI-OCT revealed a dome-shaped elevation of the choroid with effacement of the choroidal vessels, and subretinal fluid accumulation. The green arrow shows the location of horizontal section of OCT. 
intrachoroidal mass lesion, with no evidence of calcification within the mass (Figure 2B). Enhanced depth imaging-optical coherence tomography (EDI-OCT) revealed the presence of a dome-shaped elevation of the choroid with effacement of the choroidal vessels, and subretinal fluid accumulation over the mass, including the macula (Figure 2C).

Fluorescein angiography showed hypofluorescence during the early phase of the angiogram, with late leakage. Indocyanine green angiography showed hypofluorescence, which was persistent throughout the late phase of the angiogram (Figure 3). A brain/orbit magnetic resonance imaging (MRI) and computed tomography (CT) did not detect any mass lesion in his left eye or any signs of neurosarcoidosis. Systemic positron emission tomography (PET)-CT suggested there were no malignant lesions in his body, including his eyes.

Slit lamp and fundus examination revealed no sign of anemic retinopathy or intraocular inflammation such as conjunctival nodules, keratic precipitates, iridocyclitis, abnormality of the angle, vitreous opacity, vitreitis, retinal hemorrhage, exudate, or vasculitis in either of his eyes. The best-corrected visual acuity was 20/20, and intraocular pressure was within normal limits in both of his eyes.

In August 2017, which was 1 month after his first visit, he underwent posterior sub-Tenon triamcinolone acetonide $(20 \mathrm{mg})$ injection in his left eye for persistent accumulation of subretinal fluid and a choroidal mass. After topical $4 \%$ xylocaine instillation, triamcinolone acetonide was injected using a blunt cannula through a tiny conjunctival incision at $6 \mathrm{~mm}$ from the inferior-nasal limbus. Systemic steroid was decreased up to $10 \mathrm{mg}$ at the time of the injection due to the quick recovery of his platelet count back to within the normal range. After the administration of the sub-Tenon steroid injection, the subretinal fluid was quickly absorbed and the mass lesion gradually shrunk (Figure 4). Intraocular pressure stayed within the normal
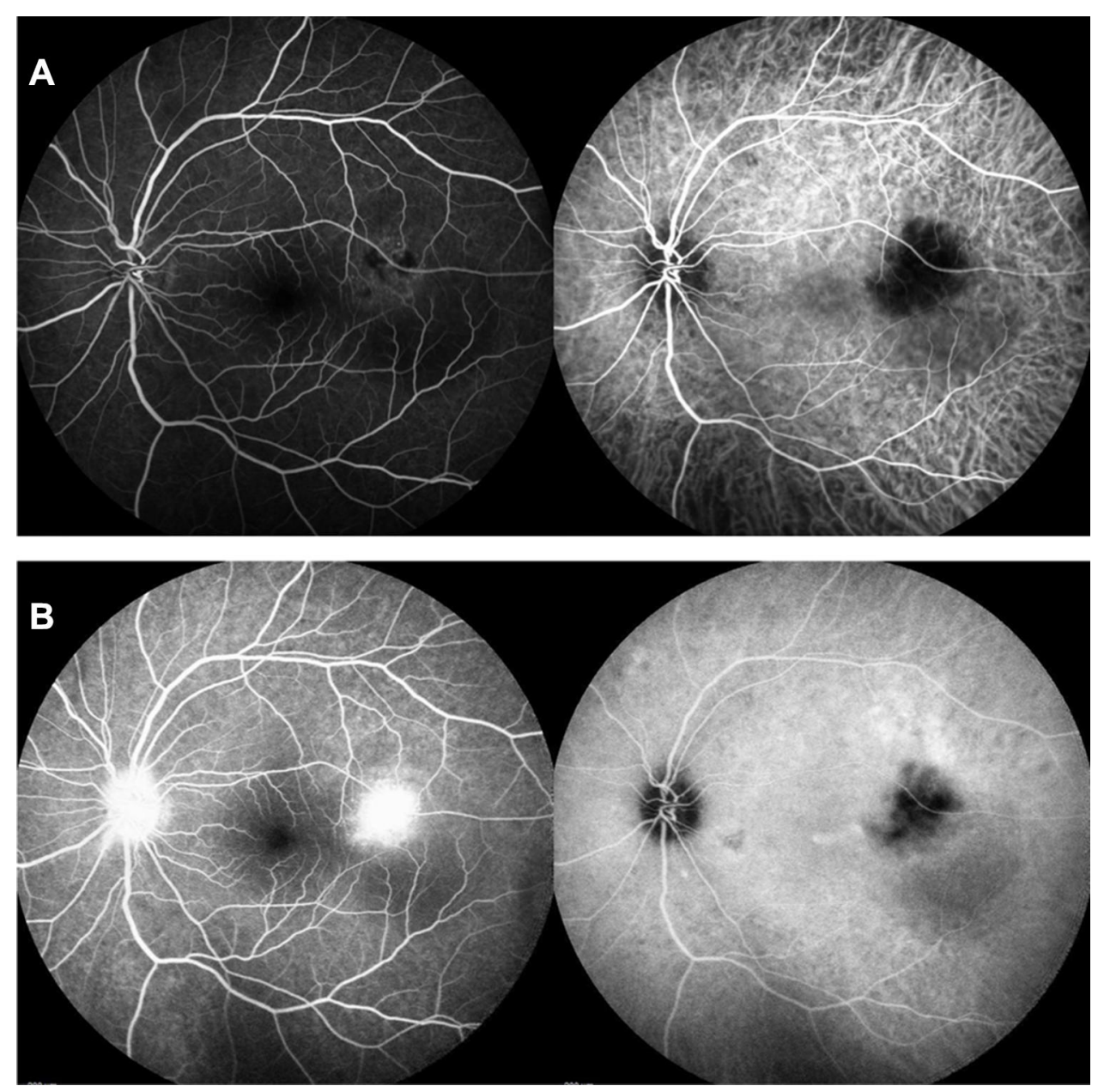

Figure 3 Fluorescein angiography (left) showed hypo-fluorescence in early phase (A) with late leakage (B). Indocyanine green angiography (right) showed hypo-fluorescence throughout the late phase $(\mathbf{B})$. 

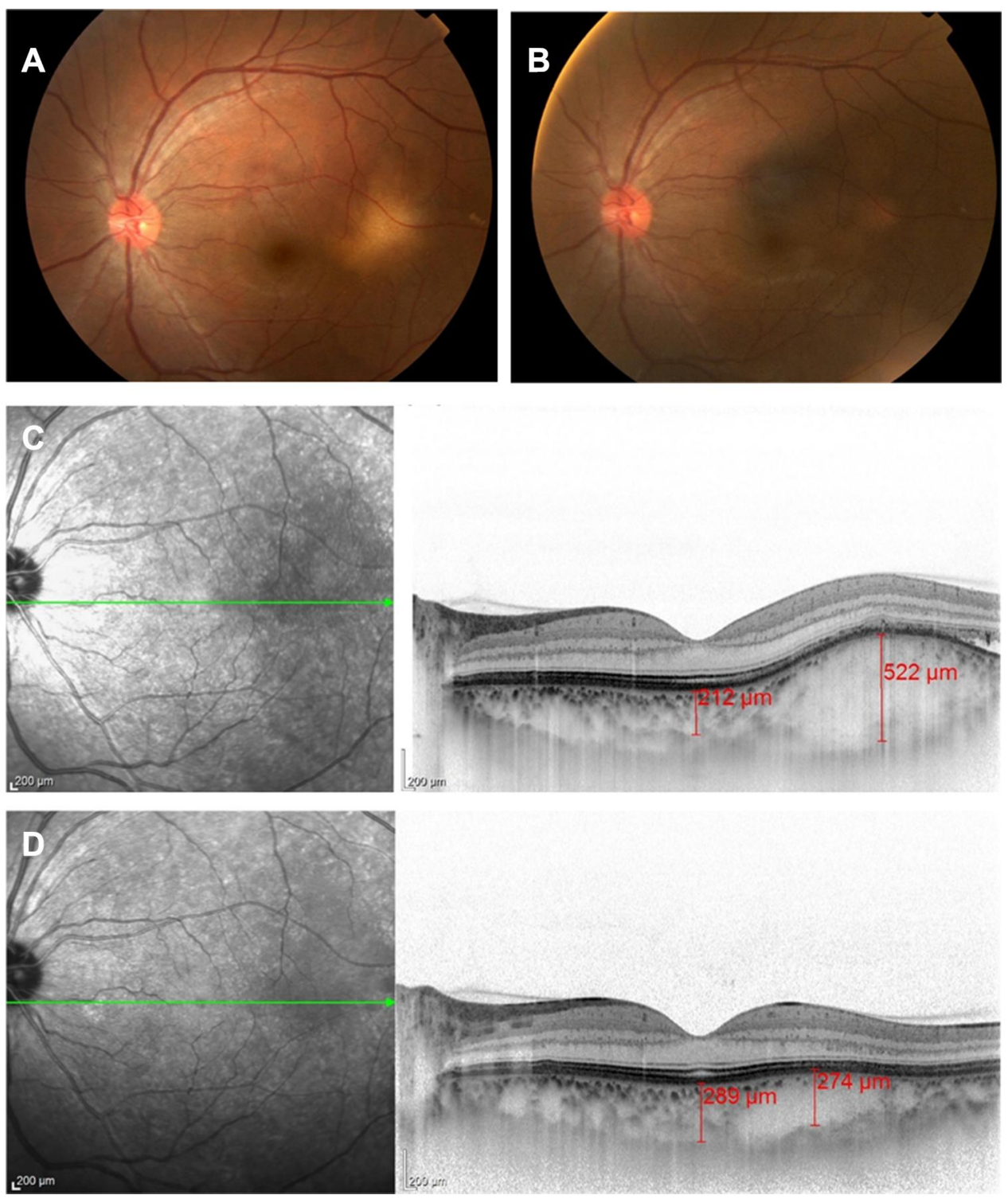

Figure 4 Subretinal fluid was absorbed and mass lesion was shrunk after the administration of the sub-tenon steroid injection. Fundus photograph at 2 weeks (A) and 28 months (B) after the injection. Enhanced depth imaging-OCT at 2 weeks (C) and 10 months (D) after injection. The green arrow shows the location of the horizontal section of OCT.

range, and visual acuity was maintained in both eyes over the next 34 months. Additional sub-tenon injections of steroid were not necessary in the patient.

\section{Discussion}

A white choroidal mass associated with retinal detachment was found incidentally in a biopsy-proven sarcoid patient who was being treated with systemic steroid for thrombocytopenia.

Choroidal granuloma associated with systemic sarcoidosis is a relatively rare occurrence. ${ }^{5-7}$ Furthermore, when attempting to determine a differential diagnosis of solitary choroidal mass, issues such as choroidal osteoma, choroidal hemangioma, amelanotic nevus, solitary idiopathic choroiditis, malignant melanoma, metastatic tumor, as well as infectious diseases such as tuberculosis, toxoplasmosis, and toxocariasis, in addition to sarcoidosis, need to be ruled out. In our current case, choroidal osteoma was ruled out, as the US-B scan indicated there was no shadowing, ${ }^{8}$ which demonstrated there was no evidence of calcification within the mass. In addition, the presence of a white color does not fit for either choroidal hemangioma ${ }^{9}$ or malignant melanoma. ${ }^{10}$ The OCT findings also were not consistent with amelanotic nevus, ${ }^{11}$ or solitary idiopathic choroiditis. Based on the initial findings, a systemic examination was then carried out and 
found to be negative for infectious diseases, with the exception for sarcoidosis. Steroid-induced exudative retinal detachment was ruled out because of the existence of the choroidal mass and the findings of the fundus angiography. Thus, the lesion was initially thought to be a possible metastatic choroidal tumor rather than sarcoid granuloma, as it was first discovered during the high dose systemic steroid administration without any ocular inflammatory findings. However, a subsequent PET-CT examination was negative for any malignancy.

Choroidal granuloma related to systemic sarcoidosis has been shown to respond well to oral corticosteroids. ${ }^{5,-12-14}$ However, care should be taken in order to avoid risks of systemic and/or ocular side-effects. Repeated intravitreal triamcinolone acetonide has also been reported to be effective for choroidal granuloma in sarcoidosis. ${ }^{7}$ Even so, it also carries some risks including endophthalmitis, hemorrhage, cataract, retinal detachment, and elevation of intraocular pressure. Ishihara et $\mathrm{al}^{15}$ reported on the efficacy of a subtenon injection of steroid for a recurrent case of choroidal granuloma in sarcoidosis after systemic steroid treatment. Kumar et al $^{16}$ reported the efficacy of the use of sub-tenon's steroid for a case of choroidal granuloma in ocular sarcoidosis with panuveitis. Moreover, the administration of a sub-tenon injection of steroid results in much fewer systemic or ocular side-effects as compared to the other routes of administration. The most common complication is an elevation of intraocular pressure, however, it was not observed in either of the previous reports.

In our current case, diagnostic and therapeutic subtenon injection of triamcinolone acetonide was performed once, after which there was complete resolution of the subretinal fluid accumulation and of the scarring of the choroidal mass without any complications or the recurrence of granuloma over the next 34 months. Based on these results, we diagnosed the choroidal mass lesion as choroidal granuloma associated sarcoidosis.

Further studies will be necessary in order to definitively clarify the efficacy of sub-tenon injection of steroid as compared to the use of systemic or intravitreal steroid for the management of choroidal granuloma in sarcoidosis.

\section{Conclusion}

Sub-tenon injection of triamcinolone acetonide can be considered for treating choroidal granuloma associated with sarcoidosis, however large scale studies are warranted.

\section{Patient Consent}

Written consent to publish this case has been obtained.

\section{Acknowledgment}

The authors wish to thank Dr Kohei Fujita for providing the histological image and advising the pathological diagnosis of sarcoidosis.

\section{Funding}

There is no funding to report.

\section{Disclosure}

The authors report no conflicts of interest for this work.

\section{References}

1. Pasadhika S, Rosenbaum JT. Ocular Sarcoidosis. Clin Chest Med. 2015;36(4):669-683. doi:10.1016/j.ccm.2015.08.009

2. Rothova A. Ocular involvement in sarcoidosis. $\mathrm{Br} J$ Ophthalmol. 2000;84(1):110-116. doi:10.1136/bjo.84.1.110

3. Atmaca LS, Atmaca-Sonmez P, Idil A, Kumbasar OO, Celik G. Ocular involvement in sarcoidosis. Ocul Immunol Inflamm. 2009;17 (2):91-94. doi:10.1080/09273940802596526

4. Ohara K, Okubo A, Sasaki H, Kamata K. Intraocular manifestations of systemic sarcoidosis. Jpn J Ophthalmol. 1992;36(4):452-457.

5. Desai UR, Tawansy KA, Joondeph BC, Schiffman RM. Choroidal granulomas in systemic sarcoidosis. Retina. 2001;21(1):40-47. doi:10.1097/00006982-200102000-00007

6. Obenauf CD, Shaw HE, Sydnor CF, Klintworth GK. Sarcoidosis and its ophthalmic manifestations. Am J Ophthalmol. 1978;86 (5):648-655. doi:10.1016/0002-9394(78)90184-8

7. Chan WM, Lim E, Liu DT, Law RW, Lam DS. Intravitreal triamcinolone acetonide for choroidal granuloma in sarcoidosis. $\mathrm{Am}$ J Ophthalmol. 2005;139(6):1116-1118. doi:10.1016/j.ajo.2004.11.055

8. Shields CL, Shields JA, Augsburger JJ. Choroidal osteoma. Surv Ophtahmol. 1988;33(1):17-27. doi:10.1016/0039-6257(88)90069-0

9. Shields CL, Hanaver SG, Shields JA, Cater J, Demirci H. Choroidal hemangioma: clinical manifestations and factors predictive of visual outcome in 200 consecutive cases. Ophthalmology. 2001;108 (12):2237-2248. doi:10.1016/S0161-6420(01)00812-0

10. Shields CL, Manalac J, Das C, Ferguson K, Shields JA. Choroidal melanoma: clinical features, classification, and top 10 pseudomelanomas. Curr Opin Ophthalmol. 2014;25(3):177-185. doi:10.1097/ICU.0000000000000041

11. Shields CL, Manalac J, Das C, Saktanasate J, Shields JA. Review of spectral domain enhanced depth imaging optical coherence tomography of tumors of the choroid. Indian $J$ Ophthalmol. 2015;63 (2):117-121. doi:10.4103/0301-4738.154377

12. Marcus DF, Bovino JA, Burton TC. Sarcoid granuloma of the choroid. Ophthalmology. 1982;89(12):1326-1330. doi:10.1016/S0 161-6420(82)34627-8

13. Tingey DP, Gonder JR. Ocular sarcoidosis presenting as a solitary choroidal mass. Can J Ophthalmol. 1992;27(1):25-29.

14. Kobayashi T, Takai N, Sato T, et al. Case of large sarcoid choroidal granuloma treated with steroid pulse therapy. Case Rep Ophthalmol. 2020;11(1):112-119. doi:10.1159/000506437 
15. Ishihara M, Shibuya E, Tanaka S, Mizuki N. Diagnostic and therapeutic evaluation of multiple choroidal granulomas in a patient with confirmed sarcoidosis using enhanced depth imaging optical coherence tomography. Int Ophthalmol. 2018;38(6):2603-2608. doi:10.10 07/s10792-017-0720-2
16. Kumar V, Gupta T, Jain S, Chanana B. Posterior sub-Tenon's triamcinolone in choroidal granuloma due to probable ocular sarcoidosis. Oman J Ophthalmol. 2013;6(2):127-128. doi:10.4103/0974-620X.11 6660

\section{Publish your work in this journal}

The International Medical Case Reports Journal is an international, peer-reviewed open-access journal publishing original case reports from all medical specialties. Previously unpublished medical posters are also accepted relating to any area of clinical or preclinical science. Submissions should not normally exceed 2,000 words or 4 published pages including figures, diagrams and references. The manuscript management system is completely online and includes a very quick and fair peer-review system, which is all easy to use. Visit http://www.dovepress.com/testimonials.php to read real quotes from published authors. 